\title{
Microwave Activities in Taiwan
}

\author{
Ruey-Beei Wu, Huei Wang, Shyh-Jong Chung, Chung-Hsin Lu, and Chun-Hsiung Chen
}

\begin{abstract}
The recent research efforts on microwave and millimeter-wave technologies in Taiwan are presented in this paper. The scope of our researches includes passive elements, and monolithic microwave/millimeter-wave integrated circuits (MMICs), together with the device modeling, design methodology, and measurement techniques. Also, the microwave industry is browsed to give a clearer picture of the combined efforts in promoting the microwave researches and development in Taiwan.
\end{abstract}

Keywords: passive components, MMICs, RE modules, wireless communications

\section{INTRODUCTION}

$\mathrm{T}$ HE $21^{\text {st }}$ century has been well recognized a century knowledge-based economy. To encourage innovation and to cultivate professionals in science and technology have been the key to the success of the knowledge-based economy, which is especially true for Taiwan, a country lack of land, capitals, and natural resources. In view of this trend, Taiwan's government has initiated several programs to cultivate the human resources and promote the advanced technologies. Two major efforts are the Program of Promoting Academic Excellence University (PPAEU) by the Ministry of Education $(\mathrm{MoE})$ since 2000 and the National Science and Technology Program (NSTP) anchored by the National Science Council (NSC) since 1999.

In PPAEU program, hundreds of proposals have been submitted for review. Only 20-30 projects were finally approved after a rigorous selection process. One of them was entitled "Advanced Technologies for Telecommunications", jointly proposed by Prof. C. H. Chen of National Taiwan University (NTU) and Prof. S. T. Peng of National Chiao Tung University (NCTU). In NSTP, only 9 areas have been strategically selected and one is National Telecommunication Program (NTP), for which wireless communications and broadband internet are the two main subjects. For the wireless

R. B. Wu, H. Wang, and C. H. Chen are with the Department of Electrical Engineering and Graduate Institute of Communications Engineering, National Taiwan University, Taipei, Taiwan, ROC. S. J. Chung is with the Department of Communications Engineering, National Chiao Tung University, and C. H. Lu is with the Information \& Communications Labs, Industrial Technology Research Institute, HsinChu, Taiwan, ROC.

This work is supported in part by the Ministry of Education, ROC, under ME-89-E-FA06-2-4-6, and the National Science Council, ROC, under Grant NSC 93- 2752-E-002-003-PAE communications, Industrial Technology Research Institute (ITRI) plays a major role while NTU and NCTU also execute some integrated projects.

In this paper, we will briefly present the overview of the microwave activities in academia and industry. More focuses will be put on the major research activities and accomplishments at NTU, NCTU, and ITRI under the support of the aforementioned programs.

\section{MicRowaVE RESEARCHES IN ACADEMIA}

\section{A. Applied Microwave Researches Sponsored by NSC}

The NSC has been the exclusively major funding source for the academia researches in Taiwan. Through the project submission and peer review, it has constantly sponsored about 100 principal investigators (PI's) in universities yearly to execute electromagnetics (EM) related applied researches.

In 2006, NSC supported 112 PI's from 47 universities to execute 119 EM-related projects. Nearly one third of the projects are from six universities: NTU (14), NCTU (9), Taiwan University of Science and Technology (6), Sun Yat-Sen University (5), Chung Cheng University (5), and Cheng-Kung University (5), where the number in the parentheses denotes the number of projects. The areas of these projects are devices (2), circuits (18), passives and packaging (30), numerical propagations and EMC (19), antennas (39), systems (3), and RF measurement and others (8)

\section{B. Microwave Researches under PPAEU}

The PPAEU at NTU and NCTU was aimed to develop various key technologies in microwave and millimeter-wave (MMW) regime. The major research topics cover passive components and antennas, high frequency monolithic integrated circuits and device modeling, and integration and packaging techniques.

The follow-on Phase II Program, supported by NSC for another four year (April 2004 to March 2008), continues the development effort and focuses on all aspects of the technologies required for the $5 / 60-\mathrm{GHz}$ dual-mode broadband wireless networks (DMBWN), including the antennas and passive components, semiconductor technologies and monolithic microwave integrated circuits (MMICs), plus the innovative packaging techniques. Moreover, the project also plans to integrate all the components and demonstrate an innovative wireless communication system offering very 
high data rates above $500 \mathrm{Mbps}$ using the $5 / 60 \mathrm{GHz}$ ISM band.

\section{1) Research Highlights at NTU}

In Phase I of PPAEU, the microwave team at NTU successfully expanded the design, analysis, integration techniques and measurement capabilities from X-band $(\sim 10 \mathrm{GHz})$ to W-band $(110 \mathrm{GHz})$. Various key components for transceiver modules with a comprehensive coverage of MMICs, passive components, antennas, and module integration have been developed and can be referred in the review paper [1].

In Phase II program, more technology breakthroughs have been developed for the RF module for DMBWN. The feasibility of the proposed architecture has been verified and submitted to IEEE 802.15.3c standard meeting for approval [2],[3]. The switched-beamformer antenna arrays are also developed. In semiconductor devices and IC area, MMW monolithic integrated circuits using bulk Si-based technologies have been demonstrated, including several state-of-the-art CMOS amplifiers, oscillators, and mixers [4]-[6], and an integrated 60-GHz transmitter composed of $\mathrm{VCO}$, mixer, power amplifier, and antenna on a single chip in SiGe BiCMOS technology [7]. Various miniaturized filters featuring multi-order spurious mode suppression and multiple passbands have been proposed [8]-[11]. Also new guiding structures for $\mathrm{RF}$ SoC and SiP are proposed [12]-[13].

The development efforts also supported the radio telescope "Array for Microwave Background Anisotropy" (AMiBA) system [14]-[15], which is currently constructed by the Department of Physics of NTU and Academia Sinica Institute of Astronomy and Astrophysics. Several MMIC chips, including the LNA, mixer and analog multipliers [16]-[18], as well as a Wband waveguide image-reject high pass filter [19] for the telescope system have been developed and currently deployed in Hawaii for field operation.

In addition, NTU have been supported from NTP to develop the key components of MMW front-end from the beginning of the program, and successfully demonstrated a $38-\mathrm{GHz}$ communication system, and a $40-48-\mathrm{GHz}$ UWB (ultra-wide-band) system. The demonstrations include $\mathrm{A} / \mathrm{V}$ signals, as well as the high speed digital data communications. The system demonstrations and module assembly have been made possible through the collaboration with Chung-Shan Institute of Science and Technology of Taiwan.

\section{2) Research Highlights at NCTU}

There are two main parts of recent microwave researches in NCTU: microwave filters and periodic structure. The studies of the microwave filters fall into three categories: planar filter with spurious suppression, planar filters with a dual-passband response, and miniaturized filter by using the low temperature co-fired ceramics (LTCC) process [20]-[25].

The researches of periodic structure include the structure developed for circular polarization selection [26] and systematically establishing the correlation between the scattering and dispersion characteristics [27] of a 2D periodic structure to identify the physical consequences of wave interaction within. In addition, the $2 \mathrm{D}$ periodic structure with defect having the frequency-selective transmission characteristic was interpreted thoroughly by the leaky parallel-plate-like waveguide mode [28].

The PPAEU has successfully upgraded the academia capabilities in various related key technologies for next generation communication systems, especially for MMW frequency. These research results will have significant impact for future research work in this area. Such upgrades can be fully reflected by the substantial increase in the number of publications in prestige IEEE journals. Taiwan has been ranked world number two in the total papers published on IEEE Transaction on Microwave Theory and Techniques in 2003 [29]. The same ranking is also reported in a recent survey on the total number of submitted and accepted papers on IEEE Microwave and Wireless Components Letters in 2005 [30].

\section{MAJOR INDUSTRIAL RESEARCHES}

Among the several research institutes for technology development, Information and Communications Research Laboratories (ICL) of ITRI has been instrumental in fostering the development of the wireless/RF communications industry. It produced the first crop of professional RF designers in Taiwan and seeded the industry development. ICL pioneered the LTCC and multi-layer ceramic in the early 1990s. Both material system and process design were out sourced to then Material Research Laboratory of ITRI. Design rules and prototype designs of filters, diplexers, chip antennas, PA modules, and Bluetooth modules are developed. The technologies were later transferred to the industry. Presently, ICL turns its attention to millimeter wave technologies on CMOS, exemplified by the $60 \mathrm{GHz}$ slot antenna.

The technology development in ICL of ITRI is briefed in the following.

\section{A. Antenna Design Technology}

Antenna in package is a highly integrated RF solution for wireless communications user terminal design. ICL engineers adeptly utilized the heat dissipating element in RF module packaging and take the effort of miniaturization one step further with boosted antenna gain through the concept of optical lens design with metamaterial [31], [32].

\section{B. Component and Module Technology}


As a communication protocol matures, design effort shifts to the end systems, namely the application part. Front end module is getting modularized, and its design is frozen in the benefit of cost cutting. The ICL engineers together with Win Semiconductor, using E/D pHEMT process, have integrated LNA, PA and RF switches into a single chip. Since passive component takes up a great portion of an RF IC die area, concurrent efforts have been put into the development of passive component modeling for III-V semiconductor process. As a result, a dual mode WiFi/WiMAX FEM with antenna, filters, T/R switch, PA and LNA into a $11 \times 11$ $\mathrm{mm}^{2}$ module has been developed [33], [34].

\section{MMW Technology}

The CMOS millimeter wave antenna is ICL's contribution to a collaborative effort with NTU. Due to the lossy substrate of the CMOS process, antenna design at MMW frequencies faces tough challenges. One of the reasonable structures is the slot antenna fed by a coplanar waveguide. The $60 \mathrm{GHz}$ antenna is manufactured by using of the TSMC 0.13 um CMOS process, with $\sim 10 \mathrm{GHz}$ antenna impedance bandwidth $(56.1-66.1 \mathrm{GHz}$, defined by $10 \mathrm{~dB}$ return loss) [35].

\section{OVERVIEW OF MICROWAVE INDUSTRY}

\section{A. Power Amplifier Modules}

The industry provides various PAs for the wireless communication applications. The major vendors are $\mathrm{RF}$ Integrated Corp. for $802.11 \mathrm{a} / \mathrm{b} / \mathrm{g}$ WLAN, CDMA, GSM/ GPRS, PHS and Bluetooth, Yuantonix's for GSM/GPRS adapter cards and devices, and Epic Comm. for 802.11 WLAN. For base station, Global PCS provides high linearity PA modules for CDMA/TDMA BTS and customer built RF Transceiver.

\section{B. Passive Circuits and Modules}

Packaging technologies with various substrates have been established to produce miniaturized and highly integrated products with high precision and long term stability. Typical solutions include thin-film modules by Cyntec, LTCC multilayer devices by Advanced Ceramic X and Maglayers, and high-density FR4 Bluetooth modules by Delta Electronics.

In parallel with $\mathrm{SoC}$ efforts, crucial technology for system in package with embedded PCB components is developed. For example, Boardtek together with Uniplus and ChipBond developed new materials for resistors and miniaturized capacitors. Advanced Semiconductor Engineering (ASE) was the first to introduce stacked CSP and multi-package ball grid array technologies in 2001. Its integrated substrate process provides optimal electrical performance for high density design requirements.

\section{CMOS RF ICS}

The CMOS RF IC technology development is blessed with the well developed semiconductor industry infrastructure and supply chain. In CMOS RF IC foundry, we have TSMC and UMC. ASE and SPIL offer excellent RF IC packaging and testing services. Giga Solution Tech provides RF device modeling.

Not all RF IC design houses devote themselves exclusively to RF IC. For example, MediaTek derives the majority of their revenue from multim edia products. Other fabless RF IC design houses include Ralink Technology, Airoha Technology, Realtek Semiconductor, Uniband Electronic, HiMARK Technology, and AlfaPlus Semiconductor, providing the chipset solutions of comprehensive coverage for various wireless communication applications.

\section{MMW Technology}

The sector of MMW industry is small but the cluster is complete. Products and services of passive components, active ICs, measurement as well as system integration all can be found in Taiwan. For example, with more than 19 years of experience in designing and manufacturing highly integrated RF transceivers, Microelectronics Technology Inc. is a supplier of microwave components and subsystems. And Airwave Technologies Inc. is specialized in broadband wireless audio and video product design.

In the component side, Universal Microwave Technology provides products for filters, diplexers, directional couplers, antennas, and all types of passive components up to $50 \mathrm{GHz}$, Allis Comm. Co. and SmartAnt are both involved in the design and manufacture of wide array of antenna products. Win Semiconductors is a GaAs foundry with HBT, HEMT, discrete and $\mathrm{MMIC}$ technologies. Chipbond and TriCOME have capability in MMW IC packaging. CMS of ITRI provides measurement services and WavePro provides measurement equipments.

\section{SUMMARY}

In this paper, we have presented the recent microwave and MMW activities in Taiwan. Due to the PPEAU and NTP programs, we have successfully promoted various related key technologies for next generation's communication systems from around 10 $\mathrm{GHz}$ to $170 \mathrm{GHz}$ in Taiwan in the past six years. These research results will have significant impact for future research work in this area.

\section{REFERENCES}

[1] H. Wang, R. B. Wu, H.-C. Lu, T.-H. Chu, P. Hsu, S.-S. Lu, and C. H. Chen, "Research on microwave and millimeter-wave technologies at National Taiwan University," in Proc. 2004 Asia-Pacific Microwave Conf., Delhi, India, Nov. 2004. 
[2] R. Fisher, "CFI Response Dec," in IEEE 802 Standard Conf., IEEE 802.15-05/439/r7, Jan. 2006.

[3] H.-J. Li, Y.-Z. Wang. Z.-H. You, W.-F. Tsen, and T.-W. Huang, "Propagation model and channel measurement for 60 $\mathrm{GHz}$ indoor wireless communication," in IEEE 802 Standard Conf., IEEE 802.15-06/0145rl, Mar.2006.

[4] R.-C. Liu, T.-P. Wang, L.-H. Lu, H. Wang, S.-H. Wang, and C.-P. Chao, "An $80 \mathrm{GHz}$ traveling-wave amplifier in a $90 \mathrm{~nm}$ CMOS technology," in 2005 Int. Solid-State Cir. Conf., pp. 154-155, San Francisco, Feb. 2005.

[5] C.-S. Lin, P.-S..Wu, H.-Y. Chang, and H. Wang, "A 9-50-GHz Gilbert-cell down-conversion mixer in $0.13-\mu \mathrm{m}$ CMOS technology," IEEE Microwave Wireless Comp. Lett., vol. 16, pp. 293-295, May 2006.

[6] C.-M. Lo, C.-S. Lin, and H. Wang, "A Miniature V-band threestage cascode low noise amplifier in 130-nm CMOS technology," in 2006 Int. Solid-State Cir. Conf., San Francisco, CA, Feb. 2006.

[7] C.-H. Wang, Y.-H. Cho, C.-S. Lin, H. Wang, C.-H. Chen, D.-C. Niu, J. Yeh, C.-Y. Lee, and J. Chern, "A 60-GHz transmitter with integrated antenna in $0.18-\mu \mathrm{m}$ SiGe BiCMOS technology," in 2006 Int. Solid-State Cir. Conf., San Francisco, CA, Feb. 2006.

[8] C. F. Chen, T. Y. Huang, C. H. Tseng, R. B. Wu, and T. W. Chen, "A miniaturized multi-layer quasi-elliptic bandpass filter with aperture-coupled microstrip resonators," IEEE Trans. Microwave Theory Tech., vol. 53, pp. 2688-2692, Sept. 2005

[9] C.-F. Chen, T.-Y. Huang, and R.-B. Wu, "Novel compact nettype resonators and their applications to microstrip bandpass filters," IEEE Trans. Microwave Theory Tech., vol. 54, pp. 755762, Feb. 2006.

[10]_, "Design of microstrip bandpass filters with multi-order spurious modes suppression," IEEE Trans. Microwave Theory Tech., vol. 53, pp. 3788-3793, Dec. 2005.

[11] - "Design of dual- and triple-passband filters using alternately cascaded multi-band resonators," IEEE Trans. Microwave Theory Tech., vol. 54, pp. 3550-3558, Sept. 2006.

[12]H.-S. Wu and C.-K. C. Tzuang, "Artificially integrated synthetic rectangular waveguide," IEEE Trans. Microwave Theory Tech., vol. 53, pp. 2872-2881, Sept. 2005

[13]C.-C. Chen and C. K. C. Tzuang, "Synthetic quasi-TEM meandered transmission lines for compacted microwave integrated circuits," IEEE Trans. Microwave Theory Tech., vol. 52, pp. 1637-1647, Jun. 2004

[14]K. Y. Lo, et. al, "AMiBA: Array for microwave background anisotropy," in AIP Conf. Proc., vol. 586, pp. 172-177, 2001.

[15]C. T. Li, D. Kubo, C. Han, C. Chen, C. Lien, H. Wang, C. Yang, T. Chiueh, J. Peterson, M. Kesteven, and W. Wilson, "A wideband analog correlator system for AMiBA," in Proc. SPIE's Int. Symp. Astronomical Telescopes Instrument., Milli, vol. 5498, pp.455-463, Glasgow, Scotland, UK, Jun. 2004

[16]B. Tzeng, C.-H. Lian, H. Wang, Y.-C. Wang, P.-C. Chao, and C.-H. Chen, "A 1 to $17 \mathrm{GHz}$ InGaP/GaAs HBT MMIC analog multiplier and mixer with broadband input matching networks," IEEE Trans. Microwave Theory Tech., vol. 50, pp. 2564-2568, Nov. 2002

[17] Y.-J. Hwang, H. Wang, and T.-H. Chu, "A W-band subharmonically pumped monolithic GaAs-based HEMT gate mixer," IEEE Microwave Wireless Comp. Lett., vol. 14, pp. 313-315, July 2004

[18]M.-F. Lei, P.-S. Wu, T.-W. Huang, and H. Wang, "Design and analysis of a miniature W-Band MMIC sub-harmonically pumped resistive mixer," in 2004 IEEE MTT-S Int. Microwave Symp. Dig., vol. 1, pp. 235-238, Dallas, Texas, Jun. 2004

[19] A.-S. Liu, R.-B. Wu, and Y.-C. Lin, "A compact design of wband high-pass waveguide filter using genetic algorithms and full-wave finite element analysis," IEICE Trans. Electron., vol. E88-C, pp. 1764-1771, Aug. 2005.

[20]J. T. Kuo, M. Jiang and H. J. Chang, "Design of parallelcoupled microstrip filters with suppression of spurious resonances using substrate suspension," IEEE Trans. Microwave Theory Tech., vol. 52, pp. 83-89, Oct. 2004.

[21]J. T. Kuo and C. Y. Tsai, "Periodic stepped-impedance ring resonator (PSIRR) bandpass filter with a miniaturized area and desirable upper stopband characteristics," IEEE Trans. Microwave Theory Tech., vol. 54, pp. 1107-1112, Mar. 2006.

[22]J. T. Kuo, T. H. Yeh and C. C. Yeh, "Design of microstrip bandpass filters with a dual-passband response," IEEE Trans. Microwave Theory Tech., vol. 53, pp. 1331-1337, Apr. 2005.

[23]S. M. Wang, C. H. Chi, M. Y. Hsieh and C. Y. Chang, "Miniaturized spurious passband suppression microstrip filter using meandered parallel coupled lines," IEEE Trans. Microwave Theory Tech., vol. 53, pp. 747-753, Feb. 2005.

[24]C. F. Chang and S. J. Chung, "Bandpass filter of serial configuration with two finite transmission zeros using LTCC technology," IEEE Trans. Microwave Theory Tech., vol. 54, pp. 2383-2388, July 2005.

[25]K. C. Lin, C. F. Chang, M. C. Wu and S. J. Chung, "Dualbandpass filters with serial configuration using LTCC technology," IEEE Trans. Microwave Theory Tech., vol. 54, pp. 2321-2328, June 2006.

[26]I.-Y. Tarn and S.-J. Chung, "A new advance in circular polarization selective surface - A three layered CPSS without vertical conductive segments," IEEE Trans. Antennas Propag., Feb. 2007

[27]R. B. Hwang, "Relations between the reflectance and band structure of 2-D metallodielectric electromagnetic crystals," IEEE Trans. Antennas Propag., vol. 52, pp. 1454-1464, June 2004.

[28]R. B. Hwang and C. C. Hsiao, "Frequency-selective transmission by a leaky parallel-plate-like waveguide," IEEE Trans. Antennas Propag., vol. 54, pp. 121-129, Jan. 2006.

[29]M. B. Steer, "Editorial," IEEE Trans. Microwave Theory Tech., vol. 52, pp. 741-742, Mar. 2004.

[30]R. Vahldieck, "Editorial," IEEE Microwave Wireless Comp. Lett., Vol. 16, pp. 57-59, Feb. 2006

[31]C. L Tang, S. H. Yeh, "Apparatus of antenna with heat slug and its fabricating process," patent pending.

[32]H. N. Liu, et al, "Design of antenna radome composed of metamaterials for high gain," in 2006 IEEE AP-S Int. Antenna Propagat. Symp Dig., Albuquerque, NM, pp. 19-22, July 2007.

[33]Y.-L. Chen and H.-H. Lin, "Novel broadband planar balun using multiple coupled lines," in 2006 IEEE MTT-S Int. Microwave Symp Dig., pp. 1571-1574, June 2006.

[34]C.-C. Chuang and C.-L. Wang, "Design of three-pole single-tobalanced bandpass filters," in Proc. 36th European Microwave Conf., pp. 1193-1196, Sept. 2006.

[35]C. S. Wang, et al, "A CMOS direct-conversion RF front-end with on-chip antenna for $60 \mathrm{GHz}$ broadband wireless communications," in Proc. 2005 IEEE Int. Analog VLSI Workshop, Bordeaux, France. 